A. K. Dupree and A. O. Benz, Eds.

\title{
Stellar Flares and Coronal Structure
}

\author{
Manuel Güdel \\ Paul Scherrer Institut, 5232 Villigen PSI, Switzerland
}

\begin{abstract}
Coronal structure and coronal heating are intimately related in magnetically active stars. Coronal structure is commonly inferred from radio interferometry and from eclipse and rotational modulation studies. We discuss to what extent flares may be responsible for coronal structure and global observable properties in magnetically active stars.
\end{abstract}

\section{Introduction}

What are stellar coronae? The answer to this simple-minded question is deeply rooted in the interplay between magnetic field generation and energy release in stellar atmospheres. The nature of this interplay is surprisingly difficult to grasp. While observables such as coronal plasma temperatures, emission measures, electron densities, non-thermal particle densities and in cases magnetic field strengths have painstakingly been measured, monitored, and correlated, our question remains one of understanding cause and effect in a complicated array of interactions. There is little doubt that magnetic fields not only confine coronal plasma but also heat this plasma once they interact due to shearing and twisting as a consequence of surface convective motions. Coronal heating thus intimately depends on magnetic structure; conversely, magnetic reconnection determines the geometry of radiating magnetic structures.

\section{Coronal Structure: Magnetospheres, Flares, Active Regions}

\subsection{Radio Interferometric Imaging of Stellar Coronae}

Radio Very Long Baseline Interferometry (VLBI) has produced some of the best evidence of stellar coronal structure related to non-thermal electrons around active stars, on milliarcsecond (mas) scales. Since the electrons are trapped in magnetic fields, they outline their overall structure. The achievable resolution is still only of order of a stellar diameter for nearby active stars or of the intrabinary distance for the closest RS CVn and Algol binary systems.

RS CVn and Algol-type binaries often reveal a bi-modal compact core + extended halo radio structure, the halo being of a total size comparable to the binary system size (e.g., Mutel et al. 1985). The extended structure is resolved in two oppositely polarized radio lobes in Algol that are aligned with the rotation axis (Mutel et al. 1998; Fig. 1b). The large-scale structure seems to be based on globally ordered magnetic fields as suggested by polarization gradients (Beasley \& Güdel 2000). Other stellar classes exhibiting large-scale 

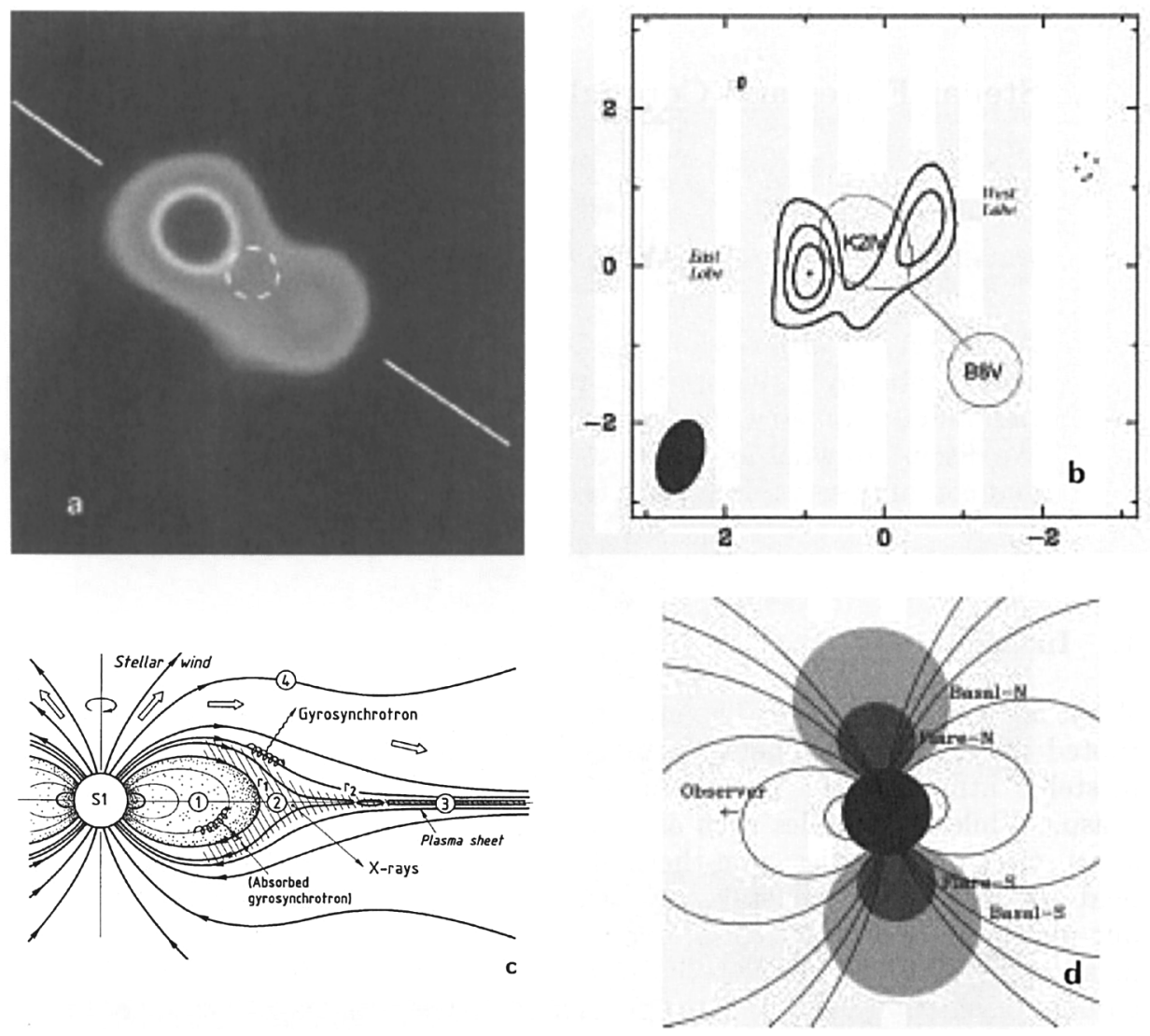

Figure 1. Radio coronal structure: Top left: VLBI image of UV Cet (resolution $\approx 0.8$ mas; after Benz et al. 1998). Top right: VLBI image of Algol (Mutel et al. 1998; units: mas). Bottom left: Model for a radio magnetosphere of a young star (André et al. 1988). Bottom right: Magnetospheric model after Mutel et al. (1998).

magnetospheres include pre-main sequence and magnetic chemically peculiar Bp/Ap stars (Phillips \& Lestrade 1988; Phillips, Lonsdale, \& Feigelson 1991, 1993; André et al. 1991, 1992; Smith et al. 2003) with sizes up to $10 R_{*}$.

The spatial and polarimetric boundary conditions are satisfied by global dipole-like magnetospheres somewhat resembling the Earth's Van Allen belts (e.g., André et al. 1988; Morris, Mutel, \& Su 1990; Linsky, Drake, \& Bastian 1992; Figure 1c). Stellar winds escaping at higher latitudes could draw the magnetic field lines into a current sheet configuration in the equatorial plane. Particles accelerated in that region travel back and are trapped in the equatorial magnetospheric cavity. Additional, dynamic ingredients may be required: The compact cores sometimes seen during enhanced flux episodes are likely to be expanding flares that evolve into extended halos where the low-density population of accelerated electrons slowly cool on time scales of days (Mutel et al. 1985).

VLBI techniques have been more demanding for dwarf stars, but spatially resolved observations of some $\mathrm{M}$ dwarfs also reveal coronae of a size much larger 
than the stellar diameter (Alef, Benz, \& Güdel 1997; Pestalozzi et al. 2000). For example, the dMe star UV Cet B is surrounded by a pair of giant synchrotron lobes, with sizes up to $2.4 \times 10^{10} \mathrm{~cm}$ and a separation of $4-5$ stellar radii along the putative rotation axis of the star, suggesting very extended magnetic structures above the magnetic poles (Benz, Conway, \& Güdel 1998; Fig. 1a). In this case, trapped high-energy electrons radiate most efficiently in the converging magnetic fields over the polar regions (see also Mutel et al. 1998 for a similar model for the magnetic field of Algol, Fig. 1d).

The prime contribution of VLBI to coronal physics has thus been in the recognition of extended, globally structured magnetic fields containing a variable number of high-energy electrons. The origin of the latter is not definitively known, and our knowledge of the connectivity of the magnetospheres to the surface magnetic fields is model-dependent.

\subsection{Eclipses}

The most productive methods of coronal structure mapping are based on (usually non-unique) image reconstructions from eclipse light curves (White et al. 1990 = W90; Siarkowsi 1992; Schmitt 1996; Güdel et al. 2003a).

Studies of extremely active binaries support a bi-modal thermal and geometric structure, somewhat reminiscent of structure inferred from VLBI and clearly unlike the non-flaring solar corona (Walter et al. $1983=$ W83; White et al. 1986 = W86; Stern et al. 1992; Ottmann 1993, 1994; Ottmann, Schmitt, \& Kürster 1993 = O93): i) Judged from rather shallow or absent X-ray eclipses in some RS CVn or Algol-type binaries, a significant fraction of the magnetically enclosed X-ray emitting plasma must be extended on scale heights $\gtrsim R_{*}$; these structures are often associated with subgiant components (e.g., in AR Lac or Algol). They seem to be predominantly very hot in X-rays ( $\gg 10 \mathrm{MK})$, reminiscent of flaring plasma. The non-detection of radio eclipses (Doiron \& Mutel 1984 for AR Lac; van den Oord et al. 1989 and Mutel et al. 1998 for Algol; Alef et al. 1997 for YY Gem) similarly suggests an extended magnetosphere also detected and resolved by VLBI $(\S 2.1)$. ii) A second type of X-ray structure found in both evolved and main-sequence binaries shows high pressure (up to several 100 dyne $\mathrm{cm}^{-2}$ ) and small, "solar-like" scale heights. Although they may be cooler than the extended structure, their pressure is reminiscent of flares.

The emission is inhomogeneous, concentrated in active regions in particular on the leading hemispheres of the stars (W90, O93) or between the stars (Culhane et al. 1990; Siarkowski 1992; Siarkowski et al. 1996; Preś, Siarkowski, \& Sylwester 1995) and at low to mid latitudes (W83, W86; W90; O93; Güdel et al. 2001a = G01a; Fig. 2e), suggesting that the "active" filling factors are modest (up to $10-20 \%$ ). The possibility that some of the material links the two stars (Fig. 2c) opens up speculations on magnetic reconnection between the two coronae (see, e.g., Uchida \& Sakurai 1983). Such has also been suggested from radio eclipse observations of the Algol-type binary V505 Sgr (Gunn et al. 1999) and the pre-cataclysmic binary V471 Tau (Lim, White, \& Cully 1996).

It is not a priori clear in which type of structure large flares occur. For $\mathrm{M}$ dwarfs, hydrodynamic simulations of large flares suggest active region sizes that cover a substantial fraction of the stellar surface (Reale et al. 2003). VLBI results suggest that flaring evolves from compact to extended structures, and this 

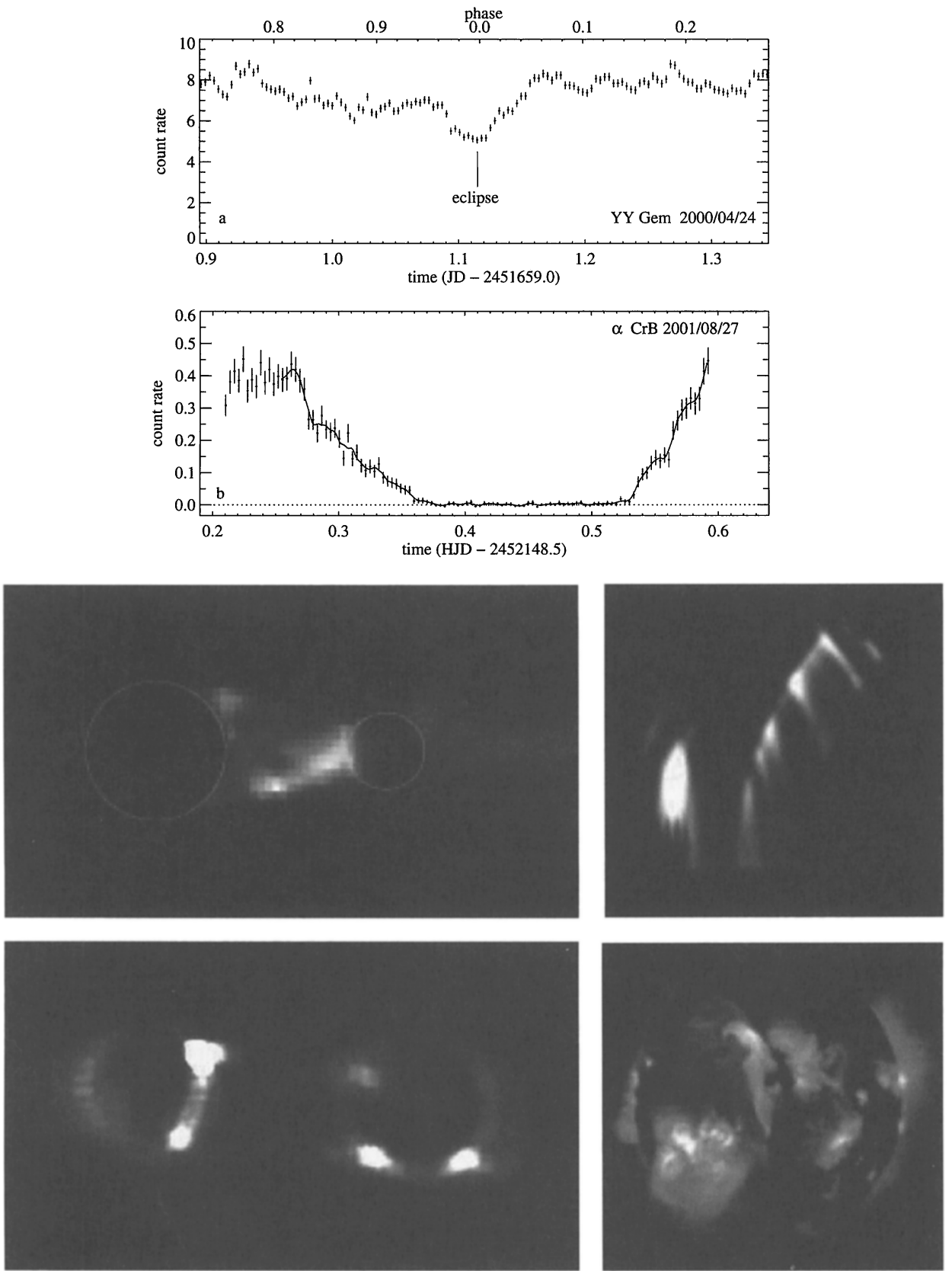

Figure 2. Stellar X-ray eclipses and map reconstructions: (a) YY Gem eclipse (G01a); (b) $\alpha$ CrB eclipse (Güdel et al. 2003a). Bottom, clockwise from upper left: AR Lac corona (Siarkowski et al. 1996); $\alpha$ CrB corona (Güdel et al. 2003a); solar image for comparison (Yohkoh mission of ISAS, Japan); YY Gem corona (phase $\approx 0.875$, G01a). 
seems to be supported by eclipse observations of flares on VW Cep and Algol that indicate relatively compact emission sites with sizes of $\approx 0.85 R_{*}$ (near one pole, Choi \& Dotani 1998 ), $\approx 0.6 R_{*}$ (scale height, near one pole, Schmitt \& Favata 1999) and $0.1 R_{*}$ (Schmitt, Ness, \& Franco 2003). Densities of $5 \times 10^{10}$ several times $10^{11} \mathrm{~cm}^{-3}$ are found, similar to solar flares.

While the above examples are all extremely active stars, a transition to more solar-like behavior is expected as the activity declines. A particularly favorable configuration is offered by $\alpha \mathrm{CrB} \mathrm{B}$, an intermediately old ( $\approx 500 \mathrm{Myr}$ ) solar analog that is totally eclipsed by an X-ray dark A star. Image reconstructions from light curves indicate a patchy structure with compact active regions, revealing electron densities up to a few times $10^{10} \mathrm{~cm}^{-3}$ but no evidence for an extended corona (Schmitt \& Kürster 1993; Güdel et al. 2003a; Fig. 2).

\subsection{Rotational Modulation}

In the absence of eclipses, a more general approach to coronal structure is provided by monitoring of rotational modulation. The Sun often shows rather pronounced X-ray rotational modulation as a small number of active regions alternately rotate into and out of view. However, rotational modulation is exceptional among stars, one of the main reasons being that most of the brightest examples are highly active; such stars are probably covered with many active regions, and intense flaring often covers up low-amplitude modulations. Such problems have hampered identification of periodic X-ray modulation in the prototypical young active star, AB Dor (White et al. 1996 and references therein; Güdel et al. 2001b; but see Kürster et al. 1997), although radio observations reveal two emission peaks per rotation seen repeatedly over long time intervals. They probably relate to preferred active longitudes (Lim et al. 1992).

Somewhat less active stars may be better-suited targets. EK Dra, a young, active solar analog, shows rotational modulation both in X-rays and radio (Güdel et al. 1995). The depth and length of the modulation constrains the X-ray coronal height and the electron densities $\left(>3 \times 10^{10} \mathrm{~cm}^{-3}\right)$ and demonstrates that much of the emitting material is concentrated in extended "active regions".

The X-ray luminosity is also below its possible maximum for stars in the "supersaturation regime" where the X-ray luminosity decreases with increasing rotation velocity. The cause is not clear, but a deep modulation of the supersaturated young star VXR45 suggests that extreme activity in these stars is not due to complete coverage of the surface with active regions (Marino et al. 2003).

Among evolved stars, the RS CVn binary HR 1099 (K1 IV + G5 V) has consistently shown X-ray/EUV rotational modulation, with a maximum at phases when the larger K subgiant is in front (Agrawal \& Vaidya 1988; Drake et al. 1994; Audard, Güdel, \& Mewe 2001). The rotationally modulated material can therefore be located - in contrast to results from eclipsing studies described above - on the $\mathrm{K}$ star hemisphere that faces away from the companion (Audard et al. 2001, using information from Ayres et al. 2001, see §2.4).

\subsection{Doppler Information}

Doppler information from X-ray spectral lines may open up new ways of imaging stellar coronae as they rotate, or as they orbit around the center of gravity in binaries. First attempts are encouraging although the instrumental limitations 
are severe. Ayres et al. (2001) find Doppler shifts in lines of HR 1099 that clearly agree with the line-of-sight velocity of the subgiant $\mathrm{K}$ star. Periodic line broadening in YY Gem, on the other hand, suggests that both components are similarly X-ray luminous (G01a). Huenemoerder et al. (2003) find Doppler motions in AR Lac to be compatible with coronae on both companions, close to the photospheric level. For the contact binary 44i Boo, Brickhouse et al. (2001) find periodic line shifts, and from the amplitudes and rotational modulation (Brickhouse \& Dupree 1998), they conclude that two dominant X-ray sources are present, one very compact and one extended, both located close to the stellar pole on the larger companion (see also Brickhouse, these proceedings).

\section{Energy Release and Coronal Structure}

The study of coronal structure confronts us with several problems that are difficult to explain by scaling (up or down) of solar coronal structure: i) Characteristic coronal temperatures increase with increasing magnetic activity (Schrijver, Mewe, \& Walter 1984; Schmitt et al. 1990; Güdel et al. 1997). ii) Characteristic coronal densities increase analogously, at least in main-sequence stars (Güdel et al. 2001b; Ness et al. 2001, 2002). iii) The maximum X-ray luminosity exceeds the level expected from complete coverage of the surface with solar-like active regions by up to an order of magnitude (Vaiana \& Rosner 1978). iv) Radio observations reveal persistent high-energy non-thermal electrons in magnetically active stars even if the lifetime of such a population should only be tens of minutes to about an hour under coronal conditions (Güdel 2002). Several of these features are reminiscent of flaring, as are some structural elements in stellar coronae $(\S 2)$. The remainder of this paper is devoted to the hypothesis that coronal structure is intimately related to explosive energy release, i.e., flares.

\subsection{Correlations between Coronal Emissions}

The time-averaged power from optical U-band flares correlates linearly with the low-level, "quiescent" X-ray luminosity (Doyle \& Butler 1985; Skumanich 1985). The latter itself correlates with the rate of X-ray flares exceeding a given energy threshold (Audard et al. 2000). Such would be expected if quiescent emission were physically related to the overall flaring rate in the active corona for example if the detected X-ray flares represented the "tip of the iceberg", the iceberg being a distribution of flares of various amplitudes, the ocean being the combined emission of a large number of superimposed small flares.

Güdel \& Benz (1993) discuss a global relation between non-thermal radio luminosities of active stars and their "quiescent" X-ray luminosities. Since the lifetime of $\mathrm{MeV}$ electrons in coronal magnetic fields requires frequent acceleration, a possible explanation again involves stochastic flares: the flare-accelerated electrons could themselves act as the heating agents via chromospheric evaporation. The smoking gun comes with the observation that the total radio and X-ray outputs of solar flares follow the same correlation (Benz \& Güdel 1994).

Since i) flares heat plasma, ii) increase the electron densities, therefore iii) increase the luminosity per surface area, and iv) amply produce non-thermal electrons, could it be that the observed phenomenology and structure on magnetically active stars is due to an ensemble of stochastic flares? 


\subsection{Coronal Variability: Quiescent versus Flares?}

Suggestive evidence has been gathered from light curves. A strong correlation between $\mathrm{H} \gamma$ flare flux and simultaneous low-level X-ray flux in dMe stars suggests that a large number of flare-like events may be responsible for the low-level emission (Butler et al. 1986). At least in main-sequence stars, there is little evidence for constant emission in high-sensitivity X-ray data (Audard et al. 2003). Frequent faint, flare-like X-ray fluctuations (Fig. 3b) are often accompanied - in fact slightly preceded - by optical (U band) bursts, the latter being a signature of the initial bombardment of the chromosphere by high-energy electrons (Güdel et al. 2002). Stochastic chromospheric evaporation could thus be the process that enriches the corona with plasma. In any case, any successful model of coronal structure and heating must account for the observed continuous variability.

\subsection{Coronal Flare Statistics}

The suggestion that stochastically occurring flares may be largely responsible for coronal heating is known as the "microflare" or "nanoflare" hypothesis in solar physics (Parker 1988). Observationally, it is supported by evidence for the presence of numerous small-scale flare events occurring in the solar corona at any time (e.g., Lin et al. 1984). Their distribution in energy is a power law,

$$
\frac{d N}{d E}=k E^{-\alpha}
$$

where $d N$ is the number of flares (per unit time) with a total energy in the interval $[E, E+d E]$. If $\alpha \geq 2$, then the energy integration (for a given time interval) diverges for $E_{\min } \rightarrow 0$, i.e., by extrapolating the power law to sufficiently small flare energies, any energy release power can be attained. This is not the case for $\alpha<2$. Evidently, then, one needs to measure the energy distribution of a statistically relevant number of flares. Solar studies have repeatedly resulted in $\alpha$ values of the order of 1.6-1.8 for ordinary solar flares (Crosby, Aschwanden, $\&$ Dennis 1993), but recent studies of low-level flaring suggests $\alpha=2.0-2.6$ (Krucker \& Benz 1998; Parnell \& Jupp 2000).

Relevant stellar studies have been rare. Early results converged to $\alpha<2$ for stellar samples (Table 1). Mixing stars at different distances and with different luminosities can introduce strong statistical bias. To avoid this, Audard, Güdel, \& Guinan (1999) and Audard et al. (2000) applied a flare search algorithm to $E U V E$ light curves of individual active main-sequence stars, taking into account flare superpositions and various binning to recognize weak flares. These results indicate a predominance of relatively steep power laws including $\alpha \geq 2$.

The identification of weak flares close to the low-level emission level is an ill-defined problem. Limited signal-to-noise ratios add to the problem. Some of these complications were overcome by fully forward modeling the superposition of a statistical ensemble of X-ray or EUV flares (Kashyap et al. 2002; Güdel et al. 2003b) including an analytical formulation (Arzner \& Güdel 2003). The results of these investigations are in full agreement, converging to $\alpha \approx 2.0-2.5$ for $M$ dwarfs. If the power-law flare energy distribution extends by about 1-2 orders of magnitude below the actual detection limit in the light curves, then the entire emission could be explained by stochastic flares. The coronal heating process would thus be one solely due to time-dependent heating by flares. 
Table 1. Stellar radiative flare energy distributions

\begin{tabular}{llll}
\hline Star sample & Photon energies & $\alpha$ & References \\
\hline M dwarfs & $0.05-2 \mathrm{keV}$ & $1.52 \pm 0.08$ & Collura et al. (1988) \\
M dwarfs & $0.05-2 \mathrm{keV}$ & $1.7 \pm 0.1$ & Pallavicini et al. (1990) \\
RS CVn binaries & EUV & 1.6 & Osten \& Brown (1999) \\
Two G dwarfs & EUV & $2.0-2.2$ & Audard et al. (1999) \\
F-M dwarfs & EUV & $1.8-2.3$ & Audard et al. (2000) \\
Three M dwarfs & EUV & $2.2-2.7$ & Kashyap et al. (2002) \\
AD Leo & EUV\&0.1-10 keV & $2.0-2.5$ & Güdel et al. (2003b) \\
AD Leo & EUV & $2.3 \pm 0.1$ & Arzner \& Güdel (2003) \\
\hline \hline
\end{tabular}

\subsection{Observables of Stochastic Flaring}

In order for stochastic flaring to be an acceptable coronal heating mechanism, a number of observables should be correctly reproduced. Flares develop characteristically in emission measure (EM) and temperature $T$ (fast rise to peak, slow decay). The individual flare histories cannot be resolved in the data but the statistical, time-integrated thermal histories of the ensemble of flares reflect in the time-averaged differential EM (DEM) distribution. The flares follow a relation in which the peak luminosity (or EM, hence the emitted X-ray energy) is a function of the peak $T$ (Feldman, Laming, \& Doschek 1995). For a simple flare shape, integration of this dependence over time and over the flare energy distribution yields an analytic expression for the DEM: Below the turnover, $\mathrm{DEM}=n^{2} d V / d \log \mathrm{T} \propto \mathrm{T}^{2 \tau_{\mathrm{T}} / \tau_{\mathrm{n}}}$ where $\tau_{T}$ and $\tau_{n}$ are the (e-folding) decay times of the flare $T$ and electron density, respectively $\left(\tau_{T} / \tau_{n}=0.5-2\right.$, Reale et al. 1993). Above the turnover, apart from small correction terms, DEM $\propto T^{-b(2-\alpha)}$ where $b \approx 5 \pm 1$ is derived from the Feldman et al. relation. Such DEMs are reminiscent of those found from X-ray spectroscopy (Telleschi et al., these proc).

Flares are often defined in light curves as excess emission above an envelope that itself is defined as "quiescent emission". This definition is problematic for two reasons. i) It is strongly dependent on the achieved signal-to-noise ratio. Any light curve shows quiescent characteristics at sufficiently small S/N or time resolution. ii) The superposition of stochastically occurring flares equally produces a lower-envelope emission level that is always present.

Fig. 3 shows simulations of superimposed flares with $\alpha=2.2$, compared with observations. The large flare observed on Proxima Centauri (Güdel et al. 2003c) was used as a shape template. The first example uses flare energies spread over a factor of ten only, the second uses three orders of magnitude. In the latter case, the curve has smoothed out to an extent that it is dominated by "quiescent" emission. The individual peaks are merely the peaks of the most energetic flares in the ensemble. The available stellar area may constrain the energy range of stochastic flares; in small, late-type $\mathrm{M}$ dwarfs, flaring active regions may cover a significant fraction of the surface (Reale et al. 2003), limiting the number of simultaneous flares (upper example in Fig. 3, e.g., Proxima Centauri).

Spectroscopic density measurements of a time-integrated, stochastically flaring corona should then yield values equivalent to the values derived from time-integrating the spectrum over a large flare. This is indeed the case. While 

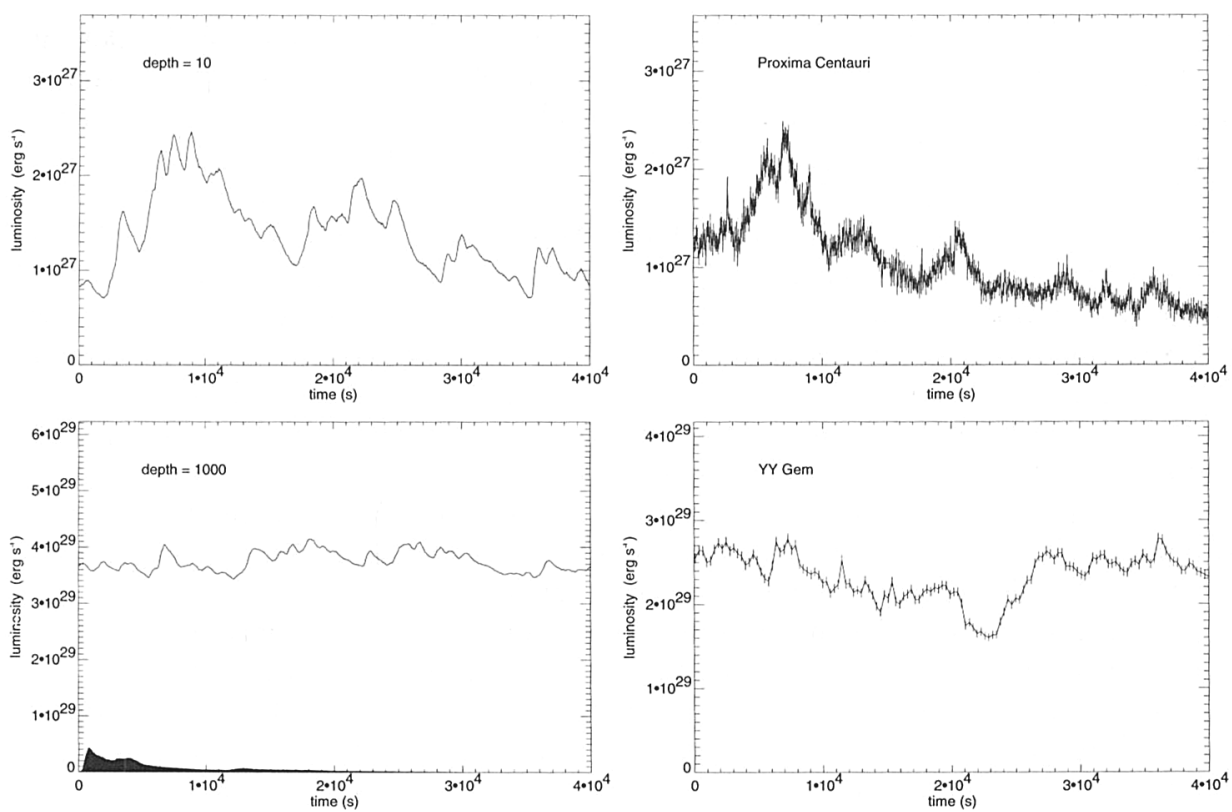

Figure 3. X-ray light curves. Left panels: Synthetic light curves from superimposed flares; largest to smallest amplitude $=10$ (upper) and 1000 (lower plot). A flare on Proxima Centauri (Güdel et al. 2002) has been used as a template, shown filled in the lower plot for the maximum amplitude contributing to the light curve. Right panels: Observed Xray light curves for comparison. Proxima Centauri (upper, Güdel et al. 2003c) and YY Gem (lower plot, including eclipse; after G01a).

large stellar X-ray flares achieve peak electron densities of several times $10^{11} \mathrm{~cm}^{-3}$, the time-integrated X-ray spectrum of the Proxima Centauri flare described in Güdel et al. $(2002,2003 \mathrm{c})$ yields a characteristic density of $\log n_{e} \approx 10.5 \pm 0.25$ derived from O VII, which compares favorably with densities found for magnetically active main-sequence stars (Ness et al. 2001, 2002).

\section{Conclusions}

Magnetically active stars frequently show bi-modal coronal structure: i) Compact "active regions" at small heights appear to be "solar-like" but often require pressures and densities comparable to solar flare pressures/densities; some compact sources have explicitly been seen to develop into expanding flares in radio VLBI observations. ii) Very extended, globally well ordered magnetic structure seen in $\mathrm{X}$-ray eclipses (as hot gas) or in radio VLBI (as a non-thermal electron population) reaches size scales of several times the stellar radius. These structures may be related to cooling, expanding "flare remnants" or large, van Allen-Belt like closed magnetospheric structures. Their thermal, density, and temporal properties are unlike typical structures in the non-flaring Sun but are reminiscent of 
flaring plasma. We have discussed expected properties of a stochastically flaring corona, such as light curves, the DEM, and the characteristic densities. While these predicted observables deviate considerably from values of the non-flaring Sun, they are reminiscent of observations of magnetically active stars.

Why should enhanced flaring occur on magnetically active stars, or in other words, why should stars with an enhanced level of magnetic field production be prone to develop flare-like magnetic coronae? We speculate that, as the magnetic dynamo increases toward more active stars, the surface filling factor of strong magnetic fields increases, increasing also the volume filling factor of closed loops in the corona. As the magnetic loops are packed closer together than on the Sun, they interact more frequently, producing a higher rate of field annihilation and reconnection, thus generating flares at a higher rate. These energy release events liberate magnetic energy and heat chromospheric plasma, evaporating it by overpressure into the magnetic loops. One thus expects both a larger average density in the corona and a higher temperature if the flare rate is higher. Increased densities and temperatures both are, in this picture, a natural consequence of a denser packing of magnetic fields in the corona, induced by a more efficient dynamo in stars with more rapid rotation. The determining factor of the coronal temperature and emission measure (hence the luminosity) is thus the flare frequency (above a certain energy threshold), which in turn is a consequence of the amount of magnetic flux production in the stellar interior.

Acknowledgments. I thank Philippe André, Arnold Benz, Robert Mutel, and Marek Siarkowski for providing figures reproduced in this paper.

\section{References}

Agrawal, P. C., \& Vaidya, J., 1988, MNRAS, 235, 239

Alef, W., Benz, A. O., \& Güdel, M. 1997, A\&A, 317, 707

André, P., Deeney, B. D., Phillips, R. B., \& Lestrade, J.-F. 1992, ApJ, 401, 667

André, P., Montmerle, T., Feigelson, E. D., Stine, P. C., Klein, K.-L. 1988 ApJ, 335,940

André, P., Phillips, R. B., Lestrade, J.-F., \& Klein, K.-L. 1991, ApJ, 376, 630

Arzner, K., \& Güdel, M. 2003, ApJ, in press

Audard, M., Güdel, M., Drake, J. J., \& Kashyap, V. 2000, ApJ, 541, 396

Audard, M., Güdel, M., \& Guinan, E. F. 1999, ApJ, 513, L53

Audard, M., Güdel, M., \& Mewe, R. 2001, A\&A, 365, L318

Audard, M., Güdel, M., \& Skinner, S. L. 2003, ApJ, 589, 983

Ayres, T. R., Brown, A., Osten, R. A., Huenemoerder, D. P., Drake, J. J., Brickhouse, N. S., \& Linsky, J. L. 2001, ApJ, 549, 554

Beasley, A. J., \& Güdel, M. 2000, ApJ, 529, 961

Benz, A. O., Conway, J., \& Güdel, M. 1998, A\&A, 331, 596

Benz, A. O., \& Güdel, M. 1994, A\&A, 285, 621

Brickhouse, N. S., \& Dupree, A. K. 1998, ApJ, 502, 918

Brickhouse, N. S., Dupree, A. K., \& Young, P. R. 2001, ApJ, 562, L75

Butler, C. J., Rodonò, M., Foing, B. H., \& Haisch, B. M. 1986, Nature, 321, 679 
Choi, C. S., \& Dotani, T. 1998, ApJ, 492, 761

Collura, A., Pasquini, L., \& Schmitt, J. H. M. M. 1988, A\&A, 205, 197

Crosby, N. B., Aschwanden, M. J., \& Dennis, B. R. 1993, Solar Phys., 143, 275

Culhane, J.L., White, N.E., Parmar, A.N., \& Shafer, R.A. 1990, MNRAS, 243, 424

Doiron, D. J, \& Mutel, R. L. 1984, AJ, 89, 430

Doyle, J. G., \& Butler, C. J. 1985, Nature, 313, 378

Drake, J. J., Brown, A., Patterer, R. J., Vedder, P. W., Bowyer, S., \& Guinan, E. F. 1994, ApJ, 421, L43

Feldman, U., Laming, J. M., \& Doschek, G. A. 1995, ApJ, 451, L79

Güdel, M. 2002, ARA\&A, 40, 217

Güdel, M., Arzner, K., Audard, M., \& Mewe, R. 2003a, A\&A, 403, 155

Güdel, M., Audard, M., Kashyap, V. L., Drake, J. J., \& Guinan, E. F. 2003b, ApJ, 582, 423

Güdel, M., Audard, M., Magee, H., Franciosini, H., Grosso, N., Cordova, F. A., Pallavicini, R., \& Mewe, R. 2001a, A\&A, 365, L344 = G01a

Güdel, M., Audard, M., Reale, F., Skinner, S. L., \& Linksy, J.L. 2003c, A\&A, in press

Güdel, M., Audard, M., Skinner, S. L., \& Horvath, M. I. 2002, ApJ, 580, L73

Güdel, M., \& Benz, A. O. 1993, ApJ, 405, L63

Güdel, M., Guinan, E. F., \& Skinner, S. L. 1997, ApJ, 483, 947

Güdel, M., Schmitt, J. H. M. M., Benz, A. O., \& Elias, N. M. 1995, A\&A, 301, 201

Güdel, M., et al. 2001b, A\&A, 365, L336

Gunn, A. G., Brady, P. A., Migenes, V., Spencer, R. E., \& Doyle, J. G. 1999, MNRAS, 304, 611

Huenemoerder, D. P., Canizares, C. R., Drake, J. J., \& Sanz-Forcada, J. 2003, ApJ, in press

Kashyap, V., Drake, J. J., Güdel, M., \& Audard, M. 2002, ApJ, 580, 1118

Krucker, S., \& Benz, A. O. 1998, ApJ, 501, L213

Kürster, M., Schmitt, J. H. M. M., Cutispoto, G., \& Dennerl, K. 1997, A\&A, 320,831

Lim, J., Nelson, G. J., Castro, C., Kilkenny, D., \& van Wyk, F. 1992, ApJ, 388, L27

Lim, J., White, S. M., \& Cully, S. L. 1996, ApJ, 461, 1009

Lin, R. P., Schwartz, R. A., Kane, S. R., Pelling, R. M., \& Hurley, K. 1984, ApJ, 283, 421

Linsky, J. L., Drake, S. A., \& Bastian, T. S. 1992, ApJ, 393, 341

Marino, A., Micela, G., Peres, G., \& Sciortino, S. 2003, A\&A, in press

Morris, D. H., Mutel, R. L., \& Su, B. 1990, ApJ, 362, 299

Mutel, R. L., Lestrade, J.-F., Preston, R. A., \& Phillips, R. B. 1985, ApJ, 289, 262

Mutel, R. L., Molnar L. A., Waltman, E. B., \& Ghigo, F. D. 1998, ApJ, 507, 371

Ness, J.-U., et al. 2001, A\&A, 367, 282

Ness, J.-U., Schmitt, J. H. M. M., Burwitz, V., Mewe, R., Raassen, A. J. J., van der Meer, R. L. J., Predehl, P., \& Brinkman, A. C. 2002, A\&A, 394, 911 
Osten, R. A., \& Brown, A. 1999, ApJ, 515, 746

Ottmann, R. 1993, A\&A, 273, 546

Ottmann, R. 1994, A\&A, 286,L27

Ottmann, R., Schmitt, J. H. M. M., \& Kürster, M. 1993, ApJ, 413, $710=093$

Pallavicini, R., Tagliaferri, G., \& Stella, L. 1990, A\&A, 228, 403

Parker, E. N. 1988, ApJ, 330, 474

Parnell, C. E., \& Jupp, P. E. 2000, ApJ, 529, 554

Pestalozzi, M. R., Benz, A. O., Conway, J. E., \& Güdel, M. 2000, A\&A, 353, 569

Phillips, R. B., \& Lestrade, J.-F. 1988, Nature, 334, 329

Phillips, R. B., Lonsdale, C. J., \& Feigelson, E. D. 1991, ApJ, 382, 261

Phillips, R. B., Lonsdale, C. J., \& Feigelson, E. D. 1993, ApJ, 403, L43

Preś, P., Siarkowski, M., \& Sylwester, J. 1995, MNRAS, 275, 43

Reale, F., Güdel, M., Peres, G., \& Audard, M. 2003, A\&A, submitted

Reale, F., Serio, S., \& Peres, G. 1993, A\&A, 272, 486

Schmitt, J. H. M. M. 1996, in Stellar Surface Structure, IAU Sym. 176, eds. K. G. Strassmeier \& J. L. Linsky (Dordrecht: Kluwer), 85

Schmitt, J. H. M. M., Collura, A., Sciortino, S., Vaiana, G. S., Harnden, F. R. Jr., Rosner, R. 1990, ApJ, 365, 704

Schmitt, J. H. M. M., \& Favata, F. 1999, Nature, 401, 44

Schmitt, J. H. M. M., \& Kürster, M. 1993, Science, 262, 215

Schmitt, J. H. M. M., Ness, J.-U., \& Franco, G. 2003, A\&A, in press

Schrijver, C. J., Mewe, R., \& Walter, F. M. 1984, A\&A, 138, 258

Siarkowski, M. 1992, MNRAS, 259, 453

Siarkowski, M., Preś, P., Drake, S. A., White, N. E., \& Singh, K. P. 1996, ApJ, 473,470

Skumanich, A. 1985, Aust. J. Phys., 38, 971

Smith, K., Pestalozzi, M., Güdel, M., Conway, J., \& Benz, A. O. 2003, A\&A, 406, 957

Stern, R. A., Uchida, Y., Tsuneta, S., \& Nagase, F., 1992, ApJ, 400, 321

Uchida, Y., \& Sakurai, T. 1983, in Activity in Red Dwarf Stars, eds. M. Rodonò \& P. Byrne (Dordrecht: Reidel), 629

Vaiana, G. S., \& Rosner, R. 1978, ARA\&A, 15, 363

van den Oord, G. H. J., Kuijpers, J., White, N. E., van der Hulst, J. M., \& Culhane, J. L. 1989, A\&A, 209, 296

Walter, F. M., Gibson, D. M., \& Basri, G. S. 1983, ApJ, 267, $665=$ W83

White, N. E., Culhane, J. L., Parmar, A. N., Kellett, B. J., \& Kahn, S., van den Oord, G. H. J., \& Kuijpers, J. 1986, ApJ, 301, $262=$ W86

White, N. E., Shafer, R. A., Horne, K., Parmar, A. N., \& Culhane, J. L. 1990, ApJ, 350, $776=\mathrm{W} 90$

White, S. M., Lim, J., Rucinski, S. M., Roberts, C., Kilkenny, D., Ryan, S. G., Prado, P., \& Kundu, M. R. 1996, in Astrophysics in the extreme ultraviolet, eds. S. Bowyer \& R. F. Malina, (Dordrecht: Kluwer), 165 